\title{
Recurrent microblazar activity in Cygnus X-1?
}

\author{
G. E. Romero ${ }^{1,2, \star}$, M. M. Kaufman Bernadó ${ }^{1}$, and I. F. Mirabel ${ }^{2,3, \star}$ \\ ${ }^{1}$ Instituto Argentino de Radioastronomía, C.C.5, (1894) Villa Elisa, Buenos Aires, Argentina \\ ${ }^{2}$ CEA/DMS/DAPNIA/Service d'Astrophysique, Centre d'Études de Saclay, 91191 Gif-sur-Yvette, France \\ 3 Instituto de Astronomía y Física del Espacio/CONICET, C.C. 67, Suc. 28, Buenos Aires, Argentina
}

Received 11 July 2002 / Accepted 20 August 2002

\begin{abstract}
Recurrent flaring events at X- and soft gamma-ray energies have been recently reported for the galactic black hole candidate Cygnus X-1. The observed fluxes during these transient outbursts are far higher than what is observed in "normal" episodes. Here we suggest that the origin of this radiation is non-thermal and produced by inverse Compton interactions between relativistic electrons in the jet and external photon fields, with a dominant contribution from the companion star field. The recurrent and relatively rapid variability could be explained by the precession of the jet, which results in a variable Doppler amplification.
\end{abstract}

Key words. X-ray binaries - stars: individual: Cyg X-1 - gamma-rays: observations - gamma-rays: theory

\section{Introduction}

Cygnus $\mathrm{X}-1$ is the most extensively studied black hole candidate in the Galaxy. It is a very bright X-ray binary with a compact object of $\sim 10.1 M_{\odot}$ and a companion O9.7 Iab star of $\sim 17.8 M_{\odot}$ (Herrero et al. 1995), at an estimated distance of $\sim 2 \mathrm{kpc}$ (e.g. Gierliński et al. 1999 and references therein). As in other sources of this type, the X-ray emission switches between soft and hard states, being most of the time in the latter. The spectrum in both states can be approximately represented as the sum of a blackbody plus a power law with exponential cut-off (e.g. Poutanen et al. 1997). During the soft state the blackbody component is dominant and the power law is steep, with a photon spectral index $\Gamma \sim 2.8$ (e.g. Frontera et al. 2001). During the hard state more energy is in the power law component, which is then harder, with photon indices $\sim 1.6$ (e.g. Gierliński et al. 1997).

The blackbody component is usually understood as emission from a cold, optically thick accretion disk, whereas the power law component is thought to be originated in an optically thin hot corona by thermal Comptonization of disk photons (Poutanen et al. 1997; Dove et al. 1997). The hot corona would fill the inner few tens of gravitational radii around the black hole. The accretion disk penetrates only marginally in the coronal region. In the hard state the thermal X-ray emission is dominated by the corona, with typical luminosities of a few times $10^{37} \mathrm{erg} \mathrm{s}^{-1}$. In the soft states, the disk approaches to the black hole and then most of the energy dissipation occurs

Send offprint requests to: G. E. Romero,

e-mail: romero@venus.fisica.unlp.edu.ar

* Member of CONICET. through it (Poutanen et al. 1997; see also Poutanen \& Coppi 1998).

Cygnus X-1 has a persistent, mildly variable, compact continuum counterpart of flat spectrum (e.g. Pooley et al. 1999). During many years, evidence for non-thermal radio jets in Cygnus X-1 was lacking, despite the efforts of the observers (e.g. Martí et al. 1996). Finally, the jet was detected by Stirling et al. (2001) at milliarcsecond resolution using VLBA observations. The jet-like feature extends up to $\sim 15$ mas with an opening angle of less than 2 degrees. The spectum seems to be flat, and no counterjet is observed. The total radio emission at $8.4 \mathrm{GHz}$ is $\sim 11 \mathrm{mJy}$, with variations of $\sim 2 \mathrm{mJy}$ over timescales of 2 days (Stirling et al. 2001). The average angle with the line of sight, if the jet is perpendicular to the disk, seems to be $\sim 30^{\circ}$ (Fender 2001).

Very recently, the Interplanetary Network detected a transient soft-gamma ray event from the general direction of Cygnus X-1 (Golenetskii et al. 2002). Analysis of previous data indicates that at least other two events were observed during 1995. These latter events were also detected by BATSE instrument on the Compton Gamma-Ray Observatory, suggesting that they were originated in Cygnus X-1 (Schmidt 2002). The luminosities above $15 \mathrm{keV}$ of the outbursts were in the range $1-2 \times 10^{38} \mathrm{erg} \mathrm{s}^{-1}$, much higher than the typical thermal luminosity in the hard state.

In this letter we suggest that these flaring events can be interpreted in terms of non-thermal microblazar activity (Mirabel \& Rodríguez 1999). We study the effects of the interaction of the relativistic jet with the ambient photon fields from the accretion disk, the corona, and the companion star, and we calculate the expected non-thermal contribution to the $\mathrm{keV}-\mathrm{MeV}$ 


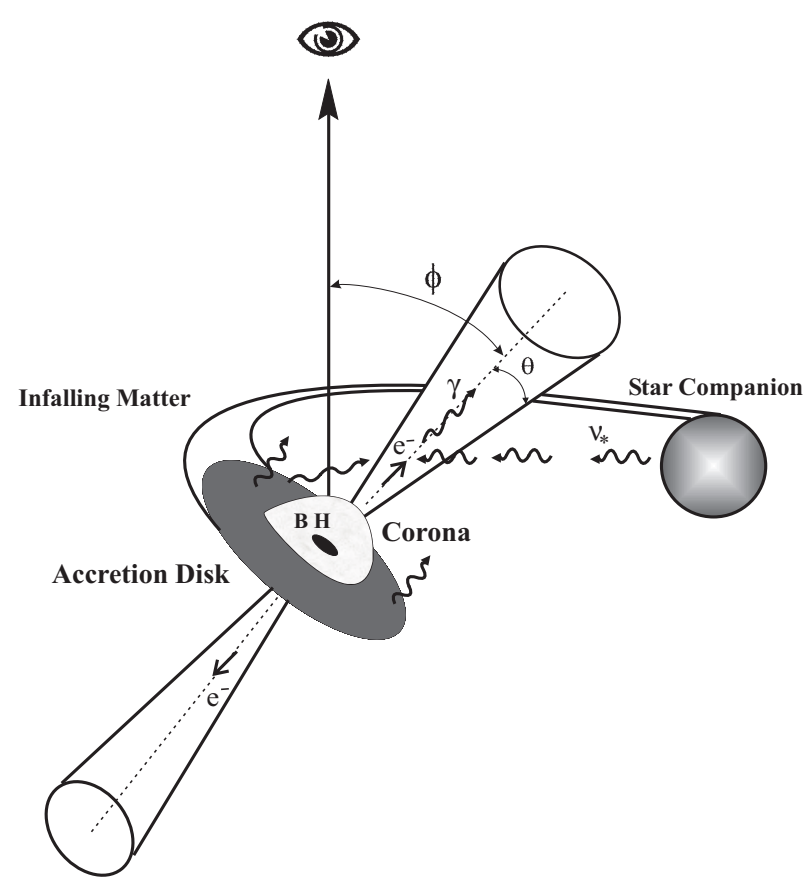

Fig. 1. Sketch of the general situation discussed in the paper. A relativistic jet is injected close to the black hole in Cygnus X-1. This jet must traverse photon fields created by the cold accretion disk, the hot corona, and the stellar companion. Inverse Compton up-scattering of some of these photons is unavoidable.

spectrum. The recurrent character of the events can be explained through variable Doppler boosting originated in the precession of the jet (Kaufman-Bernadó et al. 2002). In the next section we present the model, and then we discuss the implications.

\section{Non-thermal high-energy emission for Cygnus X-1}

We shall consider the effects of the injection of a relativistic leptonic jet at a few Schwarzschild radii from the central black hole and its subsequent propagation through the ambient photon fields (see a sketch of the situation in Fig. 1). The individual electrons have Lorentz factors $\gamma$ in the lab frame and the flow is assumed to have a bulk Lorentz factor $\Gamma$. In accordance to the disk/jet symbiosis model (e.g. Falcke \& Biermann 1999; Markoff et al. 2001) and the estimated accretion rate of Cygnus X-1 $\left(\sim 10^{-8} M_{\odot} \mathrm{yr}^{-1}\right.$, e.g. Poutanen et al. 1997), we adopt a mean particle density $n=$ $10^{14} \mathrm{~cm}^{-3}$ for the jet at $10 r_{\mathrm{s}}$ from the black hole. The electron energy distribution will be assumed to be a power law given by (in the lab frame, Georganopoulos et al. 2001): $n(\gamma)=\frac{k}{4 \pi} D^{2+p} \gamma^{-p} P\left(\gamma_{1} D, \gamma_{2} D, \gamma\right)$, where $k$ is a constant and $D=[\Gamma(1-\beta \cos \phi)]^{-1}$ is the usual Doppler factor: $\beta$ and $\phi$ are the bulk velocity in units of $c$ and the viewing angle, respectively. The function $P$ is 1 for $\gamma_{1} D<\gamma<\gamma_{2} D$ and 0 otherwise.

The jet will traverse first the coronal region. The parameters that characterize this region and its photon field in the hard state change with time. We shall assume here the typical values given by Poutanen et al. (1997) in their Table 1. All photon

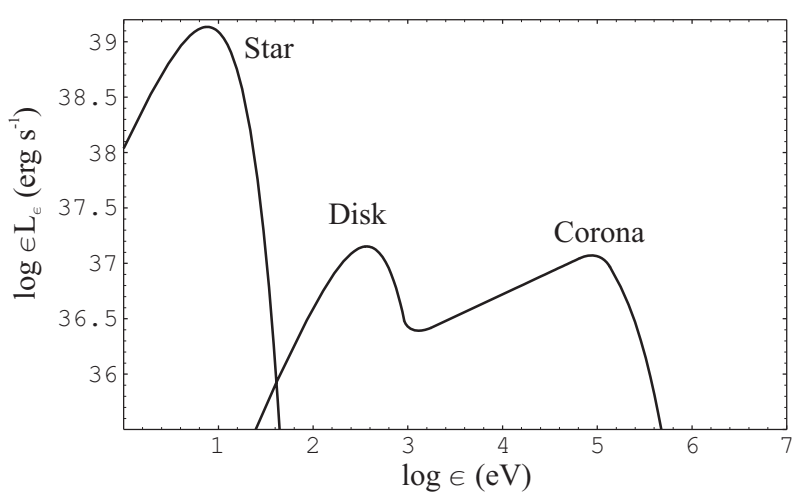

Fig. 2. External photon fields to which the jet is exposed.

fields that interact with the jet are shown in Fig. 2. The photon field of the corona is assumed to be isotropic. External inverse Compton interactions with these photons can be treated using the head-on approximation in the Klein-Nishina regime since $\gamma \epsilon_{0} \gg 1$, with $\epsilon_{0}$ the average energy of the coronal photons in units of the electron rest energy $\left(\epsilon_{0} m_{\mathrm{e}} c^{2} \sim 100 \mathrm{keV}\right)$. The reader is referred to Georganopoulos et al. (2001) for details of calculation.

In the case of the photons from the accretion disk and the companion star we can work in the Thomson regime, taken into account that the photons from the disc come from behind the jet, introducing an additional factor $(1-\cos \phi)^{(p+1) / 2}$ that reduces the effects of beaming (Dermer et al. 1992). The stellar photons, instead, can be treated as an isotropic field.

The emerging spectrum of the specific luminosity can be approximated by a power law of index $(p-1) / 2$ in the Thomson regime (Georganopoulos et al. 2001, 2002; Kaufman-Bernadó et al. 2002). When $\gamma_{2} \gg \gamma_{1}$ this can be written as:

$L_{\epsilon}=\frac{\mathrm{d} L}{\mathrm{~d} \epsilon \mathrm{d} \Omega} \approx D^{2+p} \frac{k V \sigma_{\mathrm{T}} c U 2^{p-1}}{\pi \epsilon_{0}(1+p)(3+p)}\left(\frac{\epsilon}{\epsilon_{0}}\right)^{-(p-1) / 2}$,

where $\sigma_{\mathrm{T}}$ is the Thomson cross section, $V$ is the interaction volume, and $U$ is the average energy density of the photon field. In the Klein-Nishina regime, where numerical integrations are necessary, the results significantly depart from a power law, resulting in a softer spectrum.

The Compton losses in the different regions will modify the injected electron spectrum, introducing a break in the power law at the energy at which the cooling time equals the escape time. This will occur at (e.g. Longair 1997): $\gamma_{\mathrm{b}}=$ $\left(3 m_{\mathrm{e}} c^{2}\right) /\left(4 \sigma_{\mathrm{T}} \Gamma^{2} U t_{\mathrm{esc}}\right)$. Here $t_{\mathrm{esc}}$ is average time spent by the particles in the field region (typically $t_{\mathrm{esc}} \sim l / c$, with $l$ the linear size), and the rest of the symbols have their usual meanings. The spectrum will steepen from an index $p$ to $p+1$ for energies higher than $\gamma_{\mathrm{b}}$. After the interaction of the jet with the coronal region, the modified spectrum will be injected in the stellar photon field region, suffering further losses and modifications.

In our calculations we have adopted two different values for the original electron energy index: a hard index $p=1.5$ and a steeper index $p=2.3$. The jet was assumed forming an angle of 30 degrees with the line of sight, and a bulk Lorentz factor $\Gamma=5$ was adopted. The initial part of the jet was modeled as a cylindrical structure; the coronal region, following 


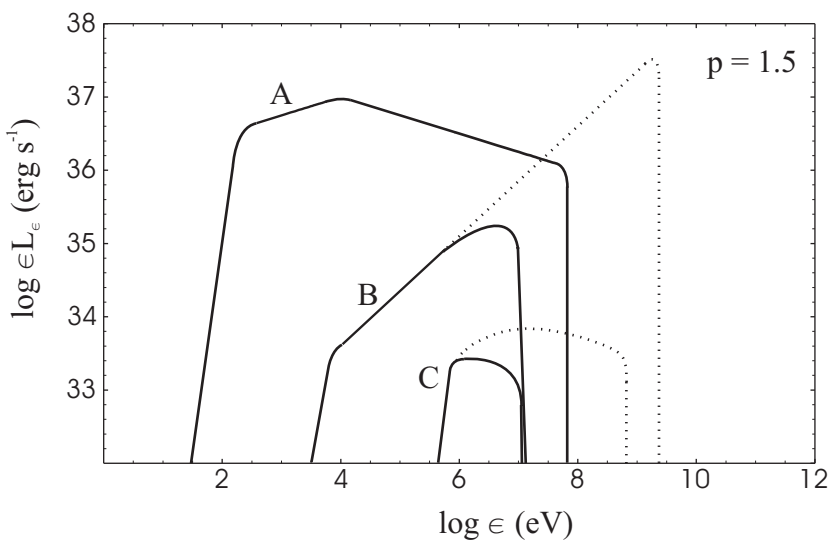

Fig. 3. Results of the model for an injection electron spectrum with index $p=1.5$ in a cylindrical jet forming a viewing angle of 30 degrees. The bulk Lorentz factor is $\Gamma=5$ and the electron power law extends from $\gamma_{1}=2$ to $\gamma_{2}=10^{3}$. Three different components are shown, resulting from the up-scattering of star (A), disk (B), and corona (C) photons. Radiation absorbed in the local photon fields is shown in dashed lines. Notice that the contribution from the coronal photons is not a power law because of the Klein-Nishina effects.

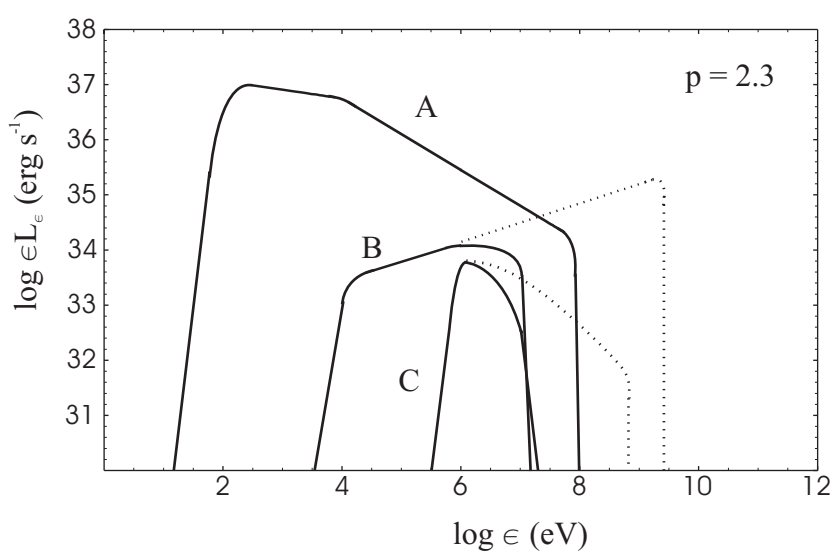

Fig. 4. Idem Fig. 3 for $p=2.3$.

Poutanen et al. (1997), was considered as a spherical region of $\sim 500 \mathrm{~km}$ in radius. Gamma-rays produced within this region will be mostly absorbed in the local field through pair creation. Using the simple formulae by Herterich (1974) along with the adopted parameters for the coronal region, we estimate an optical depth $\tau \sim 1$ for photons of $5 \mathrm{MeV}$. The probability for a $10 \mathrm{MeV}$ photon to escape from the corona is only 0.1 .

The results obtained for the different models are shown in Figs. 3 and 4. There, we show the different components due to external Compton scattering of the different photon fields in the lab frame, for the different injected electron distributions we have assumed. This non-thermal contribution should be added to the thermal components shown in Fig. 2 in order to recover the total emission. We can see that the non-thermal emission is dominated by the up-scattering of the stellar photons. Above $5 \mathrm{MeV}$ almost all energy flux comes from this component.

For a viewing angle of 30 degrees, we still have the thermal emission dominating by a factor of 5 . But if we introduce the

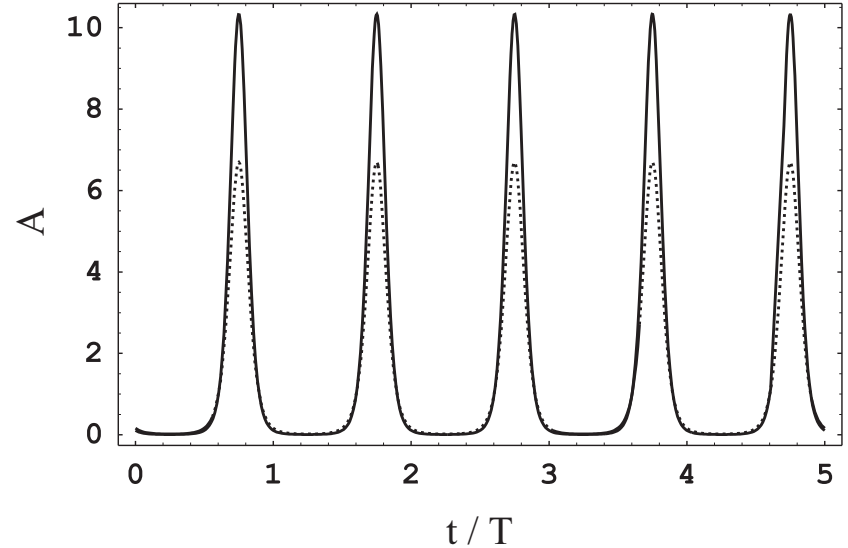

Fig. 5. Variation of the amplification factor for the external inverse Compton emission from the jet (electron power-law indices of $p=$ $1.5-$ dots - and $p=2.3$ - solid line - ) as a function of time in the precessing microblazar model for Cygnus X-1 (precessing opening angle of 16.5 degrees). Time units are normalized to the precessing period.

gravitational effects of the companion star, then precession of the disk should occur (Larwood 1998; Kaufman-Bernadó et al. 2002). In Fig. 5 we show the modification of the beaming amplification factor of the external inverse Compton emission for a precessing angle of 16.5 degrees. The time axis is normalized in units of the period $T$. We see that there is a variation of about 1 order of magnitude in the emission measured in the observer's frame because of the precession. This means that when the jet is closer to the line of sight, the non-thermal luminosity can reach values of $\sim 10^{38} \mathrm{erg} \mathrm{s}^{-1}$, as observed in the recurrent outbursts detected by the Interplanetary Network. The transit through the peak of flux magnification can be very fast in the observer's frame, leading to quick and transient states when the total flux is dominated by the non-thermal contribution.

The angular velocity of the tidally induced precession can be approximated by $\omega_{\mathrm{p}} \approx-3 G m \cos \theta / 4 r_{\mathrm{m}}^{3} \omega_{\mathrm{d}}$ (Katz 1980; Romero et al. 2000), where $\theta$ is the half-opening angle of the precession cone, $m$ is the mass of star, $r_{\mathrm{m}}$ is the binary separation, and $\omega_{\mathrm{d}}$ is the Keplerian velocity of the accretion disc, which can be written in terms of the mass $M$ of the black hole as $\omega_{\mathrm{d}}=\left(G M / r_{\mathrm{d}}^{3}\right)^{1 / 2}$. For $\theta=16.5 \mathrm{deg}$ as we have assumed, the observed orbital period of 5.6 days, and a precessing period of $\sim 140$ days (Brocksopp et al. 1999, see below), we get a disk size of $\sim 3.9 \times 10^{11} \mathrm{~cm}$, quite reasonable for a wind-accreting system like Cygnus X-1.

\section{Discussion}

The above outlined model incorporates the different known components of Cygnus X-1, accretion disk, corona, stellar companion, and relativistic jet, in an integrated picture where transient non-thermal outbursts are a natural and expected result. The amplitude of these outbursts can be similar to what has been recently observed in some intriguing flaring episodes in this source. Our model is different from the model recently proposed by Georganopoulos et al. (2002) not only because we incorporate the effects of precession, but also because we 
do not attempt to explain the bulk of X-ray emission as nonthermal all the time. This emission is normally dominated by thermal Comptonization in the hot corona around the black hole, except during the microblazar phase, and in this case we incorporate the effects of the interaction of the jet with the corona in our calculations. We emphasize that, as it is shown in Figs. 3 and 4, during the transient microblazar phase the X-and soft $\gamma$-ray spectrum will be softer than in the normal hard state, when the coronal emission dominates. This is an unavoidable consequence of the steepening produced by Compton losses in the injected electron spectrum and can be used to test our proposal, not only through new observations of Cygnus X-1, but also of other potential microblazars as LS5039 (Paredes et al. 2000).

Recently, Brocksopp et al. (1999) have found multiwavelength evidence for the presence of a $142.0 \pm 7.1$ days period in Cygnus X-1. The optical and X-ray period seem to be originated in the precession of the accretion disc (Brocksopp et al. 1999), whereas the modulation at radio wavelengths is probably produced by the associated precession of the jet (see Pooley et al. 1999). The morphology of the extended radio jet, with a clear bend, is also consistent with a precession of the inner beam (Stirling et al. 2001). The periodic signal in the radio lightcurve, however, is not expected to be as strong as at high energies since the magnification factor for the synchrotron emission goes as $D^{(3+p) / 2}$, whereas for the external Compton component it goes as $D^{2+p}$ (Georganopoulos et al. 2002).

The time lag between the two high-energy flares observed in 1995 is $\sim 75$ days, about a half of the value reported by Brocksopp et al. (1999), but since Cygnus X-1 is a windaccreting system variations in the period along a span of several years are possible. Certainly, more observations on longer time spans are necessary to constrain the dynamical models. In the model presented here the duty cycle of the blazar phase is rather small, $\sim 10 \%$. Future $\mathrm{X}$-ray observations of non-thermal flares can be used for a better determination of the geometric parameters.

An interesting feature of our model is that most of the gamma-rays produced within the coronal region will be absorbed by pair production. Sooner or later these pairs will annihilate producing a broad, blueshifted feature in the $\mathrm{MeV}$ spectrum. Details of calculation of the emerging spectrum are beyond the scope of this Letter (see Abraham et al. 2001), but it is clear that the forthcoming INTEGRAL satellite will be able to probe Cygnus X-1 spectrum and its temporal evolution at this energy range, helping to test and constrain the model here proposed. Hopefully, very soon we will be able to clarify the role played by non-thermal processes in this fascinating object.
Acknowledgements. G.E.R. is mainly supported by Fundación Antorchas. Additional support comes from the research grants PICT 03-04881 (ANPCT) and PIP 0438/98 (CONICET). He is grateful to Isabelle Grenier for useful discussions and to the staff of the Service D'Astrophysique, Centre d'Études de Saclay, where part of his research for this project was carried out. I.F.M. acknowledges support from grant PIP 0049/98 and Fundación Antorchas. This research benefited from the ECOS French-Argentinian cooperation agreement.

\section{References}

Abraham, Z., Romero, G. E., \& Durouchoux, P. 2001, ESA SP-459, 131

Brocksopp, C., Fender, R. P., Larimov, V., et al. 1999, MNRAS, 309, 1063

Dermer, C. D., Schleickheiser, R., \& Mastichiadis, A. 1992, A\&A, 256, L27

Dove, J. B., Wilms, J., Maisack, M., \& Begelman, M. G. 1997, ApJ, 487,759

Falcke, H., \& Biermann, P. L. 1999, A\&A, 342, 49

Fender, R. P. 2001, MNRAS, 322, 31

Frontera, F., Palazzi, E., Zdziarski, A. A., et al. 2001, ApJ, 546, 1027

Georganopoulos, M., Kirk, J. G., \& Mastichiadis, A. 2001, ApJ, 561, 111

Georganopoulos, M., Aharonian, F. A., \& Kirk, J. G. 2002, A\&A, 388, L25

Gierliński, M., Zdziarski, A. A., Done, C., et al. 1997, MNRAS, 288, 958

Gierliński, M., Zdziarski, A. A., Poutanen, J., et al. 1999, MNRAS, 309, 496

Golenetskii, S., Aptekar, R., Mazets, E., et al. 2002, IAUC, 7840

Herrero, A., Kudritzki, R. P., Gabler, R., et al. 1995, A\&A, 297, 556

Herterich, K. 1974, Nature, 250, 311

Katz, J. I. 1980, ApJ, 236, L127

Kaufman Bernadó, M. M., Romero, G. E., \& Mirabel, I. F. 2002, A\&A, 385, L10

Longair, M. S. 1997, High Energy Astrophysics (Cambridge University Press, Cambridge), 281

Markoff, S., Falcke, H., \& Fender, R. P. 2001, A\&A, 372, L25

Martí, J., Rodríguez, L. F., Mirabel, I. F., \& Paredes, J. P. 1996, A\&A, 306,449

Mirabel, I. F., \& Rodríguez, L. F. 1999, ARA\&A, 37, 409

Paredes, J. M., Martí, J., Ribó, M., \& Massi, M. 2000, Science, 288, 2341

Pooley, G. G., Fender, R. P., \& Brocksopp, C. 1999, MNRAS, 302, L1

Poutanen, J., Krolik, J. H., \& Ryde, F. 1997, MNRAS, 292, L21

Poutanen, J., \& Coppi, P. 1998, Phys. Scr., T77, 57

Romero, G. E., Chajet, L., Abraham, Z., \& Fan, J. H. 2000, A\&A, 360, 57

Schmidt, M. 2002, IAUC, 7856

Stirling, A. M., Spencer, R. E., de la Force, C. J., et al. 2001, MNRAS, 327,1273 